\title{
Evaluation of Resiliency of Transportation Networks After Disasters
}

\author{
Derek Freckleton, Kevin Heaslip, William Louisell, and John Collura
}

\begin{abstract}
The resiliency of infrastructure, particularly as related to transportation networks, is essential to any society. This resiliency is especially vital in the aftermath of disasters. Recent events around the globe, including Hurricane Katrina and significant seismic events in Haiti, Chile, and Japan, have increased the awareness and the importance of resiliency. Transportation systems are key to response and recovery. These systems must withstand stress, maintain baseline service levels, and be stout enough in physical design and operational concept to provide restoration to the system. Analysis of a transportation network's resiliency before a disruptive event will help decision makers identify specific weaknesses within the network so that investments and improvement projects are prioritized appropriately. Previous research in quantification of network resiliency was expanded into a proposed methodology, through which understanding and applying concepts of network resiliency could preclude many devastating effects of destabilizing events and preserve the quality of life and economic stability.
\end{abstract}

The transportation network is critical to a nation's way of life and its economic vitality (1). The world's dependence on transportation systems is growing as regional, national, and international societal interaction and economic activities become more fully integrated and interdependent. Transportation systems are needed to provide food, medicine, and mobility. Disruptions of these systems can be devastating. History has shown how important, and how fragile, the transportation network is and that far-reaching effects can be felt from network disruption.

Hurricane Katrina illustrated this in 2005. Fragility, inadvertently built into the local and regional transportation networks, was revealed when poor system performance limited evacuation and recovery efforts. In 2010, a magnitude 7.0 earthquake in Haiti devastated its capital and killed hundreds of thousands of people. Thousands more perished after the event because much-needed goods and services could not be transported. Although planes carrying aid began arriving as early as the next day, humanitarian groups struggled to get supplies to victims. Poor roads and debris made navigation nearly impossible.

According to the U.S. Geological Survey (2), the modified Mercalli intensity (MMI) scale value assigned to a specific site after an earth-

D. Freckleton, Utah State University, 4110 Old Main Hill, Logan, UT 84322. K. Heaslip, 233 Engineering Lab, Utah State University, Logan, UT 32611. W. Louisell, Utah Transportation Center, Utah State University, Logan, UT 84321. Current address for W. Louisell: 419 Barbadian Way, Mount Pleasant, SC 29464. J. Collura, University of Massachusetts, Amherst, 214C Marston Hall, Amherst, MA 01003. Corresponding author: K. Heaslip, kheaslip@engineering. usu.edu.

Transportation Research Record: Journal of the Transportation Research Board, No. 2284, Transportation Research Board of the National Academies, Washington, D.C., 2012, pp. 109-116.

DOI: 10.3141/2284-13 quake may have a more meaningful measure of severity than the earthquake's magnitude because intensity refers to the effects experienced at that place. The Haitian earthquake was assigned an MMI value of 9 , indicating severe damage to poorly built structures (fallen chimneys, smokestacks, columns, monuments, and walls; heavy furniture overturned), as well as considerable damage in specially and well-designed frame structures, which were thrown out of plumb. Substantial buildings also incurred severe damage with partial collapses and buildings shifted off their foundations (2).

Almost 1 month later, a magnitude 8.8 earthquake rocked the Chilean coast; however, it was assigned an MMI value of 8 . Despite the significantly stronger event in Chile, the MMI value was lower because damage was less significant. Whereas Haiti took months to recover, Chile recovered within weeks. The key difference between these two examples is resilience.

Resiliency, if properly understood and applied, can preclude many of the devastating effects of disasters. Resilient transportation systems may reduce the probability of failure within the system and reduce the consequences of any failure that occurs, thus improving recovery time. Understanding the resiliency of a transportation system after a disaster has occurred does little to mitigate the effects of the event. Thus, this paper expands on the conceptual framework developed by Heaslip et al. to assess the network resiliency of a system before a destabilizing event (3). This process identifies weaknesses within the network and provides decision makers with a flexible and robust method for quantifying resiliency. The information can be used to properly prioritize transportation investments to enhance network resiliency.

\section{DEFINING RESILIENCE}

The concept of resilience is broadly applied in many fields of study (e.g., engineering, psychology, sociology, economics). Similar concepts are flexibility, redundancy, reliability, elasticity, and risk management. In economics, the term "resilience" refers to the ability to recover quickly from a shock (shock counteraction), to withstand the effect of a shock (shock absorption), and to avoid the shock (vulnerability) (4). In social science, resilience is the capacity of a system that has been exposed to hazards to adapt by resisting or changing, so that it can reach and maintain an acceptable level of functioning and structure (5). In earthquake engineering, researchers define seismic resilience as the ability of social units (e.g., organizations, communities) to mitigate hazards, contain the effects of disasters, and carry out recovery activities in ways that minimize social disruption and reduce the effects of future earthquakes (6). Community seismic resilience is the capacity to absorb stress, manage it, and recover from it (7). More generally, resilience is the capacity to absorb shocks gracefully (8).

The concept of resilience has been studied in the field of transportation engineering as well. Conceptual frameworks have been created 
to define and measure resilience within the area of transportation. Transportation resilience can be defined in several ways:

- A system's ability to maintain its demonstrated level of service or to restore itself to that level of service in a specified time frame (3),

- A characteristic that enables the system to compensate for losses and allows the system to function even when infrastructure is damaged or destroyed (9),

- A system's ability to accommodate variable and unexpected conditions without catastrophic failure (10), and

- A system's ability to absorb the consequences of disruptions to reduce the impact of disruptions and maintain freight mobility (11).

The definition of resiliency used in this paper is the ability for a transportation network to absorb disruptive events gracefully and return itself to a level of service equal to or greater than the predisruption level of service within a reasonable time frame.

\section{CONCEPTUAL FRAMEWORK}

For the methodology developed by Heaslip et al. (3), the parameters for resiliency were defined within the context of a resiliency cycle, which recognizes the cycle of normalcy, breakdown, annealing, and recovery, as shown in Figure 1.

Breakdown is the measure of degradation caused by an event. An event-driven breakdown degrades the system's performance and often reduces its ability to absorb additional pressure from eventinduced demand or a follow-on disaster (such as an aftershock to an earthquake). Annealing and recovery are measures of how quickly the network can regain or exceed the level of service present before the breakdown. Once the damage has been done to the network, it will attempt to seek a new equilibrium, and system users will attempt to find pockets of unused capacity leading to an annealing phase. The annealing process is the progression of the network toward normalcy, but it may be hindered by physical damage and loss of capacity. Recovery of the network, if required to offset physical damage, is dependent on the nature of the damage and the access to goods or services needed to repair the network.

For the annealing and recovery processes, time is a critical measure of the success of a resilient system. For a network that is not resilient, delays in beginning those processes, or delays throughout, may cause the effects of the breakdown to spread to areas other than where the breakdown occurred. Consequently, slow recovery times may be devastating to the local, regional, and national economies of the affected area.

To enhance the resiliency of a network, then, one must understand the characteristics of a transportation network that affect its overall resilience. When considering critical components of transportation resiliency, Murray-Tuite identifies such characteristics as

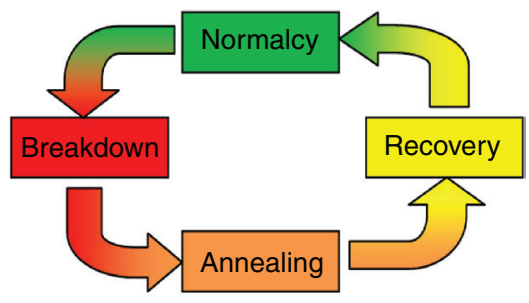

FIGURE 1 Resiliency cycle. redundancy, diversity, efficiency, autonomy, strength, adaptability, collaboration, mobility, safety, and recovery (12). The methodology developed by Heaslip et al. incorporates a dependency diagram using these, as well as other characteristics relating to transportation network resiliency (3).

\section{METHODOLOGY}

The methodology used in this paper is similar to the work of Urena Serulle et al., in which a fuzzy inference approach is used (13), but the methodology differs in that metrics used by Heaslip et al. (3) were brought back, and the following methodology was performed. The contributions of this new study are found in the specific definitions of each metric used in the calculation of total network resiliency.

At the center of the methodology are four metric groups identified by the research community: metrics related to the individual, to the community, to the economy (14), and to recovery (15).

Individual resiliency metrics show whether the transportation network provides options and utility to individual users. These options are present to meet their transportation needs even under unusual and unexpected conditions. Community resiliency metrics show whether the transportation system fulfills the needs of the community. The network can safely and efficiently accommodate unusual conditions, including construction projects, emergencies, special events, and large gatherings without major impact. Economic resiliency metrics show whether the transportation network provides services even if a particular resource, such as fuel, becomes scarce or expensive (14). Recovery metrics show whether the network can anneal and recover. The recovery metric group examines the resources and qualities necessary to restore resiliency to the network.

Each metric group is supported by a lower tier of measurements of specific attributes, and each contributes to a higher tier that can be used to compare resiliency between assessed areas or that can be used to measure the contribution of specific projects and policies to the improvement of regional resiliency.

The attributes, or variables, are measured on a qualitative scale with input values of low, medium, and high. Numeric values are assigned to each qualitative value as well, such as 1, 2, and 3, respectively. However, the inverse order may be necessary in such cases as transport cost indices and delays. High transportation costs as well as high delays relate to resiliency in poor conditions. Therefore, numeric ranges for such variables are assigned 3,2, and 1, respectively. Measurement range definitions are given in Table 1 . Each variable is weighted equally according to the number of variables feeding into its related core metric. Therefore, variables determining each core metric group are 1/5 for each individual metric group variable, $1 / 5$ for each community metric group variable, $1 / 7$ for each economic metric group variable, and 1/3 for each recovery metric group variable. The variables considered in more than one metric group will carry a more significant weight for the final total network resiliency. This is by design. For example, mode choice should carry a more significant weight than transport cost because in an extreme case, if no mode choice were available, the cost to use a mode of transport becomes meaningless.

The value for each core metric group is determined by the inputs of each variable supporting it. For the metric groups to be standardized, the numeric value of each variable $(1,2$, or 3$)$ is multiplied by its weight, and the summation of each of those values is then divided by three (because of the three input options: low, medium, and high). The outputs for each metric group, as well as the total 
TABLE 1 Variable Input Range Definitions

\begin{tabular}{lllll}
\hline Variable & Measurement & Low & Medium & High \\
\hline Mobility index & Level of service & E-F & C-D & A-B \\
Delay encountered & Travel time index & $<1.13$ & $1.13-1.15$ & $>1.15$ \\
Food medicine index & (No. of locations $/ 10,000$ people) & $\leq 2$ & $2-4$ & $\geq 4$ \\
Personal transport cost index & $(\$ / \mathrm{km})$ & $<0.40$ & $0.40-0.47$ & $>0.47$ \\
Personal mode choice & Modes of transport & $\leq 1$ & 2 & $\geq 3$ \\
Network redundancy & (Arterial kilometers $\left./ \mathrm{km}^{2}\right)$ & $<2$ & $2-5$ & $>5$ \\
Infrastructure alignment & $($ kilometers $)$ & $<5$ & $>15$ & $5-15$ \\
Goods and material access & $\left(\right.$ No. of locations $\left./ 10 \mathrm{~km}^{2}\right)$ & $<0.5$ & $0.5-1$ & $>1$ \\
Commercial mode choice & Modes of transport & $\leq 1$ & 2 & $\geq 3$ \\
Industrial mode choice & Modes of transport & $\leq 1$ & 2 & $\geq 3$ \\
Network management & Level & I-II & II-IV & IV-V \\
Fuel and energy access & $\left(\right.$ No. of locations $/ 10 \mathrm{~km}^{2}$ ) & $<3$ & $3-6$ & $>6$ \\
Commercial transport cost index & $(\$ / \mathrm{km})$ & $<0.75$ & $0.75-1.00$ & $>1.00$ \\
Industrial transport cost index & $(\$ / \mathrm{km})$ & $<0.75$ & $0.75-1.00$ & $>1.00$ \\
Emergency response & (hours) & $>2$ & $0.25-2$ & $<0.25$ \\
Resources available & Disaster response contractors & $<10$ & $10-20$ & $>20$ \\
\hline
\end{tabular}

network resiliency, consist of nine parameters and are stratified according to the following values:

- Extremely low: $0.33 \leq 0.41$,

- Very low: $0.41 \leq 0.48$,

- Low: $0.48 \leq 0.56$,

- Medium low: $0.56 \leq 0.63$,

- Medium: $0.63 \leq 0.70$,

- Medium high: $0.70 \leq 0.78$,

- High: $0.78 \leq 0.85$,

- Very high: $0.85 \leq 0.93$, and

- Extremely high: $0.93 \leq 1.00$.

For the determination of the total network resiliency, the core metric groups are each weighted equally, like the attributes, according to the number of variables feeding into the next level of the dependency diagram. Therefore, each is weighted $1 / 4$ in determining the total network resiliency. The sum of the values obtained previously for each metric group multiplied by their weight is used to determine the final qualitative value for total network resiliency. This value is also assigned according to the stratification listed previously. This final output produced by the dependency diagram represents the estimated resiliency of the network as a whole. Higher total network resiliency values signify an enhanced likelihood that the system will be able to fulfill the definitions of transportation resiliency given earlier.

After this initial network resiliency is determined, a sensitivity analysis can be performed on the network by changing the input values of any attribute that a proposed project may change in the network. A comparison of the results of this process with the initial resiliency will show which projects will most benefit the network, providing valuable information about prioritization for investments and improvement projects.

\section{APPLICATION}

An example was developed to illustrate the application of the proposed methodology. The scenario addresses a seismic event occurring along a major fault line on the Wasatch Front near Salt Lake
City, Utah. Because the MMI scale gives meaningful measures of the levels of severity of such events, it is important to understand the classification, or stratification, of that scale. The following is an abbreviated description of the 12 levels of intensity as given by the U.S. Geological Survey (2):

1. Not felt except by a few under especially favorable conditions.

2. Felt only by a few persons at rest, especially on upper floors of buildings.

3. Felt quite noticeably by persons indoors, especially on upper floors of buildings. Many people do not recognize it as an earthquake. Standing motorcars may rock slightly. Vibrations similar to the passing of a truck. Duration estimated.

4. Felt indoors by many, outdoors by few during the day. At night, some awakened. Dishes, windows, doors disturbed; walls make cracking sound. Sensation like heavy truck striking building. Standing motorcars rock noticeably.

5. Felt by nearly everyone; many awakened. Some dishes, windows broken. Unstable objects overturned. Pendulum clocks may stop.

6. Felt by all, many frightened. Some heavy furniture moved. A few instances of fallen plaster. Damage slight.

7. Damage negligible in buildings of good design and construction, slight to moderate in well-built ordinary structures. Considerable damage in poorly built or badly designed structures. Some chimneys broken.

8. Damage slight in specially designed structures, considerable damage in ordinary substantial buildings with partial collapse. Damage great in poorly built structures. Fall of chimneys, factory stacks, columns, monuments, walls. Heavy furniture overturned.

9. Damage considerable in specially designed structures, welldesigned frame structures thrown out of plumb. Damage great in substantial buildings with partial collapse. Buildings shifted off foundations.

10. Some well-built wooden structures destroyed, most masonry and frame structures destroyed with foundations. Rails bent.

11. Few if any (masonry) structures remain standing. Bridges destroyed. Rails bent greatly.

12. Damage total. Lines of sight and level are distorted. Objects thrown into the air. 


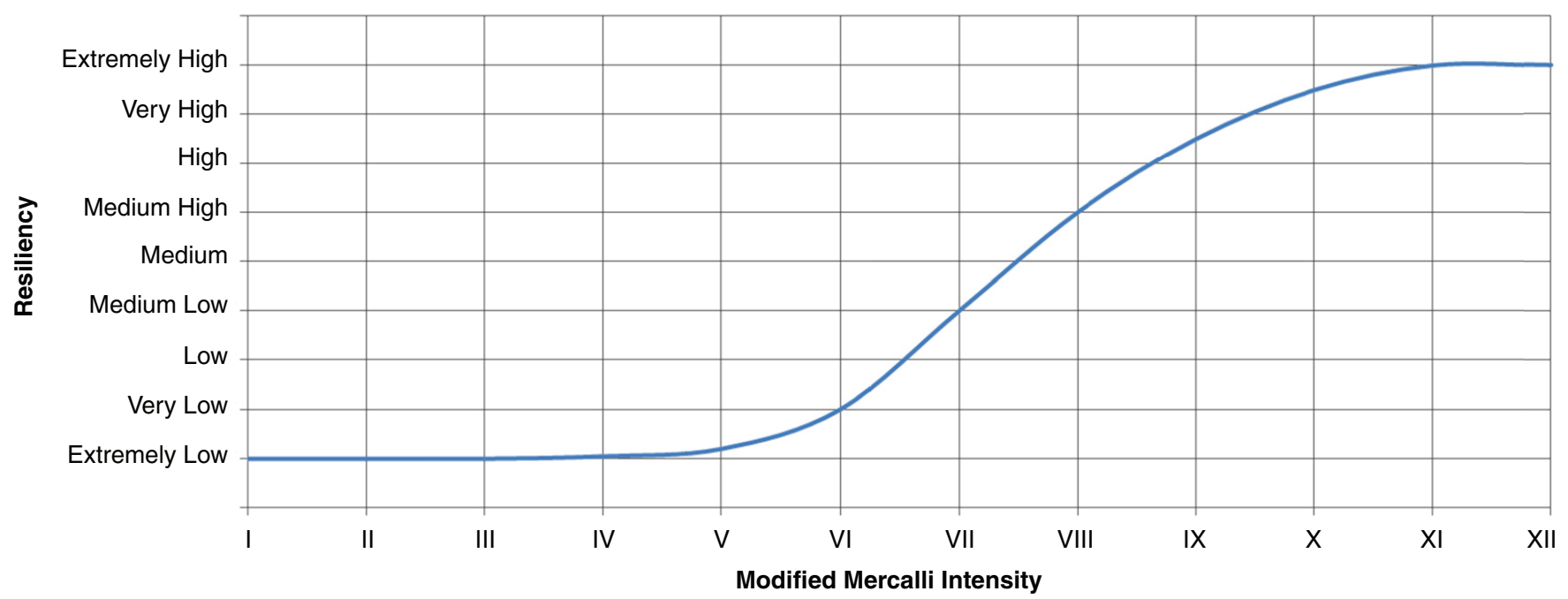

FIGURE 2 Resiliency versus MMI curve.

Figure 2 is an estimated curve for the total network resiliency necessary to withstand events of varying intensity, according to the descriptions of damage given by the MMI scale.

Research was performed at the University of Utah to assess the effects a significant seismic event could have on the economy of Salt Lake City, Utah (16). Information from that research was used to build this application, allowing a comprehensive assessment of the resiliency of Salt Lake City's transportation network. The area of study is approximately $520 \mathrm{~km}^{2}$ and has a population of about 500,000 (17). The region in which most of the damage was expected contains several major roadways, including federal and state highways, as well as railroads and the Salt Lake City International Airport.

\section{INITIAL RESILIENCY}

The dependency diagram, centered on the core metric groups, was used to determine the initial resiliency of Salt Lake City's transportation network. The results are given in Figure 3. Measures that are considered in more than one core metric group are indicated by dotted lines.

Given the input values determined through the initial assessment of Salt Lake City, a total network resiliency of high was obtained through the methodology, as shown in the figure. This value represents the approximate resiliency of the Salt Lake City network. From the curve given in Figure 2, it is estimated that high resiliency corresponds to the ability of Salt Lake City's transportation network to withstand a Level 8 event without significant disturbance.

A detailed description of each attribute in the dependency diagram is given in this section. Data were collected for the study area in relation to those descriptions, and input values were inserted into the dependency diagram according to the range definitions given in Table 1.

\section{Mobility Index}

Mobility, which refers to the movement of people or goods (10), increases resiliency by fostering annealing. It explains the demand on the infrastructure as well as its performance. Transportation engineers may use standardized methods for calculating mobility in terms of level of service, outlined in the Highway Capacity Manual (18). Level-of-service values range from high to low, A through F, respectively. From January to August 2010, the average level of service in Salt Lake City during peak periods was B (19).

\section{Delay Encountered}

Delay is an average additional amount of time that a traveler likely will experience because of a network disruption (10). Delay lengthens the time before annealing begins, particularly in the case of sudden events. As delay increases, one's ability, as well as motivation, to travel weakens. The Texas Transportation Institute defines a travel time index as a measure of congestion that focuses on individual trips and each mile of travel. It is a ratio of travel time in the peak period to travel time in free-flow traffic. For example, an index value of 1.20 would indicate that a 30-min free-flow trip would take approximately 36 min during peak periods (20). In 2007, Salt Lake City had a travel time index value of 1.19 , which was considered much higher than average in a comparison of several urban areas with populations between 500,000 and 1 million (20).

\section{Food Medicine Index}

The food medicine index represents the availability of food or medicine within the network. Increased access to food and medicine throughout the network will provide resiliency, as well as a buffer for annealing to begin without major disruption to the network. The food medicine index is measured in number of locations per capita. Such locations include hospitals and grocery stores containing pharmacies. Roughly 200 of these locations were located within the study area; therefore Salt Lake City has approximately four locations per 10,000 people.

\section{Personal Transport Cost Index}

The personal transport cost index represents the cost incurred by individuals to use the network for transportation. As transportation 


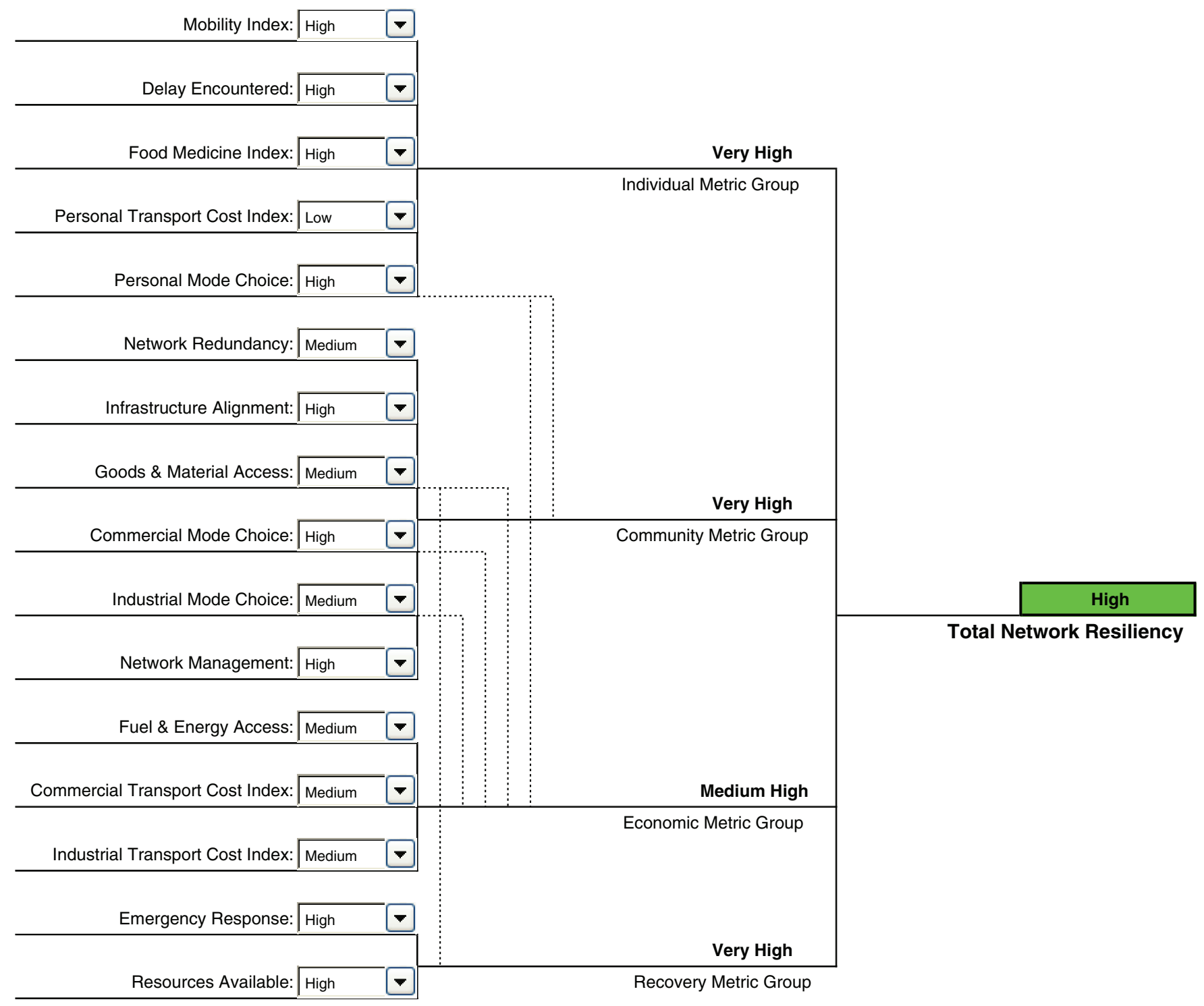

FIGURE 3 Initial resiliency.

costs increase, budgets are increasingly strained (21). Therefore, high costs for transport will limit options when individuals react to destabilizing events. As the price of fuel and other supporting commodities and services increases, one's ability as well as motivation to travel decreases. Measurements for this variable are given in units of cost per distance traveled. Values may be computed with the typical cost of travel calculator wizards and planning program components available to metropolitan planning organizations. According to the Internal Revenue Service (22), the current standard mileage rate for the use of a car, van, or pickup truck is $\$ 0.31$ per kilometer.

\section{Personal Mode Choice}

Mode choice represents the ability to use alternative modes of transportation throughout the network. Increased transportation mode choices will enhance options when individuals react to destabilizing events. If only one mode of transport remains usable after an event, many other aspects of the network (i.e., mobility, delay, and cost) would be significantly affected as individuals scramble for necessary goods and services. However, if more than one mode of transport were usable, those parameters would likely be somewhat alleviated. Therefore, personal mode choice is measured in total number of mode choices available to individuals. Salt Lake City uses personal automobile transportation and various modes of public transportation, including bus and railway.

\section{Network Redundancy}

Redundancy, representing backup organization built into a system to prevent total system failure, is a major component of network resiliency (23). Redundancy within the network reduces choke points that could limit the options for annealing to begin. It represents the ability for a traveler to adjust routes as necessary to detour around an affected section of the network. Research has shown that higher values of road density result in better developed networks because of their inherent redundancy (24). However, despite the 
road density that local roads may offer, it is unlikely that they will provide significant relief (e.g., cul-de-sacs). Thus, this variable is measured in freeway and arterial road density, or the amount of arterial lane kilometers within a specified area. Roughly 1,950 lane kilometers of freeways and arterial roads were measured within the study area.

\section{Infrastructure Alignment}

The infrastructure alignment variable represents the availability of secondary infrastructures within a network (alternative routes). A wellaligned secondary infrastructure will decrease the impact on the network of the destabilizing event. The variable is measured in proximity, or the distance between primary and secondary infrastructures. At closer distances, the event that destabilizes the primary infrastructure will likely affect the secondary infrastructure as well. Also, at further distances, travelers are less able to use the secondary infrastructure. Therefore, intermediate distances are most desired when this variable is considered. The average distance between infrastructures in Salt Lake City is approximately $6 \mathrm{~km}$, resulting in an intermediate distance, and therefore a high rating, according to Table 1.

\section{Goods and Material Access}

Good and material access represents the availability of goods and materials within the network specific to transportation needs. Access to these supplies will provide the means for annealing. Goods and materials access is measured in density, or the number of locations that can provide transportation-related goods and materials within a specified area. Examples of such locations are warehouses, lumber yards, and concrete suppliers, as well as airports and train stations because goods and materials may be transported to those locations, if they are functioning after the destabilizing event. Approximately 50 of these locations were identified within the study area; therefore Salt Lake City was found to contain less than 1 location per $10 \mathrm{~km}^{2}$.

\section{Commercial Mode Choice}

Increased mode choice for commercial transport will enhance options when commercial interests react to destabilizing events. If only one mode of commercial transport remains usable after an event, the ability to transport goods to their necessary locations may be limited. However, if more than one mode of transport were available, the ability to transport goods to their necessary locations would increase. Therefore, commercial mode choice is measured in total number of mode choices available to commercial entities. Truck, rail, and air modes are available for commercial use within the area of study.

\section{Industrial Mode Choice}

Increased mode choice for industrial transport will increase options when industrial interests react to the destabilizing event and will enhance the ability to transport industrial items to begin annealing. If only one mode of industrial transport remains usable after an event, the ability to transport necessary items to specific locations may be significantly restricted. If more modes of transport were available, the ability to transport items to affected areas would increase. Therefore, industrial mode choice is measured in the total number of mode choices available to industrial entities. Truck and railway transportation is available for industrial transport in the Salt Lake City area.

\section{Network Management}

Network management refers to the activities, methods, procedures, and tools that pertain to the operation, administration, maintenance, and provision of network systems (25). Its goal is to ensure effective, efficient, and standardized operations within and among transportation modes (1). Advanced network management provides real-time shifting of resources and demands on the network, which allows annealing to begin and dulls the impact of destabilizing events. Following are examples of network management used for the measurements shown in Table 1:

Level 1. Police officers directing traffic,

Level 2. Traffic signals,

Level 3. Dynamic traffic signal timing and ramp metering,

Level 4. Traffic cameras and variable message signs, and

Level 5. Intelligent transportation systems and advanced traveler information systems.

Salt Lake City has the highest number of traffic cameras per capita and per roadway mile of all transportation networks in the country. Because of this, along with the city's traffic management center, Salt Lake City's network management performs at a Level 5.

\section{Fuel and Energy Access}

The fuel and energy access variable represents the availability of fuel and energy within the network. Limits on access would weaken the ability of the network to anneal and would increase the impact of the destabilizing event. Fuel and energy access is measured in density, or the number of locations that provide fuel and energy within a specified area. Roughly 235 locations within the study area provide fuel and energy, with a resulting value of about 4.5 locations per $10 \mathrm{~km}^{2}$.

\section{Commercial Transport Cost Index}

The commercial transport cost index represents the cost for commercial entities to use the network for transportation. High costs for commercial transport will limit options when commercial interests react to the destabilizing event. This variable reacts similarly to the personal transport cost index, because significantly high costs for fuel and other supporting commodities and services may make commercial transport unlikely. This variable is measured in cost per distance. Commercial transport in Salt Lake City costs approximately $\$ 0.90$ per kilometer.

\section{Industrial Transport Cost Index}

The industrial transport cost index represents the cost for industrial entities to use the network for transportation. High costs for industrial transport will limit options when industrial interests react to 
the destabilizing event and will affect the ability to transport industrial items to begin annealing. As with the personal and commercial transport cost indexes, significantly high transportation costs may make industrial transport unlikely. This variable is measured in cost per distance and is approximately equal to commercial transport cost (\$0.90 per kilometer).

\section{Emergency Response}

Emergency response represents the ability of a region to mobilize response efforts without the help of other areas. According to the Federal Emergency Management Agency, the first $48 \mathrm{~h}$ can make the difference in allaying the effects of a disaster (26). Rapid response times thus will increase resiliency. This variable goes slightly beyond that time frame and assumes that considering first response, the first $2 \mathrm{~h}$ may be the most critical. It is therefore measured in the time needed for first responders to react to an event. In Salt Lake City, first-response times average $5 \mathrm{~min}$.

\section{Resources Available}

Resources available represents the availability of people, organizations, and equipment. Available resources for procuring the materials necessary to anneal increases network resiliency. The U.S. Army Corps of Engineers has developed a contractor registry to assist with disaster response (27). It uses its engineering and contracting capabilities to support the Federal Emergency Management Agency and other federal, state, and local government agencies in a variety of missions during natural and manmade disasters. Information in this registry may be used by Corps of Engineers offices searching for specific goods or services during emergencies. Therefore, this variable is measured by the number of licensed and registered disaster response contractors within the area. A total of 55 disaster response contractors that could rapidly respond to an emergency in Salt Lake City were found within this registry's records (28).

\section{SUGGESTIONS FOR IMPROVEMENT}

It is desirable to obtain the most benefits at the lowest cost for improvement projects. However, an inverse relationship typically exists for these two elements. Lower costs usually result only from the sacrifice of certain benefits, and vice versa. As resiliency is bolstered, rising to the next level becomes increasingly expensive, often with little change in overall resiliency. Therefore, decision makers should prioritize projects according to the results expected from implementation, weighing cost against overall benefit.

Some variables in the dependency diagram are considered in more than one core metric group. Thus, these variables carry more weight in consideration of the outcome. Variables that contribute to core metric groups with fewer attributes supporting them will carry heavier weight when the results are considered. For example, each variable contributing to the recovery metric group has a weight of $1 / 3$, whereas each variable contributing to the economic metric group has a weight of $1 / 7$. Hence in this case, improving variables contributing to the recovery metric group will generally have a greater effect on the results. Therefore, it is suggested that a sensitivity analysis done to determine prioritization of improvement projects give specific attention to attributes that carry more weight.
TABLE 2 Improvements and Results

\begin{tabular}{|c|c|c|}
\hline Variable & Initial Value & Improved Value \\
\hline $\begin{array}{l}\text { Improvement in goods } \\
\text { and material access }\end{array}$ & Medium & High \\
\hline \multicolumn{3}{|l|}{ Impact on } \\
\hline Community metric group & Very high & Very high \\
\hline Economic metric group & Medium high & High \\
\hline Recovery metric group & Very high & Extremely high \\
\hline Total network resiliency & High & Very high \\
\hline
\end{tabular}

\section{RESULTS}

Attributes related to mode choice and access to goods and materials were considered first for improvement. Because building a new mode choice in Salt Lake City would be expensive, goods and materials access was chosen as the first improvement project. The improvement of this attribute consequently raised the total network resiliency of the system. Subsequent to those improvements, other attributes were tested but with little or no improvement in total outcome. Therefore, boosting goods and materials access became the highest priority for improvements, if funding for improvement projects were limited. Prioritizing goods and materials improvements provided the best results for the least cost.

With the assumption that improvements to goods and materials changed the network, the new qualitative value for goods and materials access was input to the dependency diagram, and a new total network resiliency value of very high was obtained. The impact this change had on the dependency diagram is shown in Table 2.

The improved value of very high represents the approximate resiliency of Salt Lake City after the suggested improvements have been implemented. According to Figure 2, it is estimated that very high resiliency corresponds to Salt Lake City's transportation network now having the ability to withstand a Level 9 event without significant disturbance to the transportation network.

\section{CONCLUSION}

Along with an ever-growing dependence on transportation systems to provide life's essentials, societies develop a continual need to maintain the serviceability of that system, especially in case of unpredictable disaster. Resilience must be a key focus in development of new transportation systems as well as improving existing ones. Application of the proposed methodology showed that specific weaknesses may be pinpointed within a transportation network. Then, given that information, continuation of the network assessment showed that it provides a practical means of properly prioritizing improvement projects to enhance the system's total network resiliency. Because of the nature of disasters, it is difficult to predict how a system might be damaged. The best one can do is be prepared to mitigate negative results. Successfully building the resilience of a transportation network will help stabilize the economy and well-being of communities, regions, and nations. The methodology proposed in this report has been proved through analysis to provide the means to prioritize transportation infrastructure projects to successfully increase network resiliency. 


\section{REFERENCES}

1. U.S. Transportation Security Administration. Transportation Sector Network Management. 2010. http://www.tsa.gov/what_we_do/tsnm/ index.shtm. Accessed Aug. 2, 2010.

2. U.S. Geological Survey. The Modified Mercalli Intensity Scale. Oct. 27, 2009. http://earthquake.usgs.gov/learn/topics/mercalli.php. Accessed Aug. 5, 2010.

3. Heaslip, K., W. Louisell, and J. Collura. A Method to Evaluate Transportation Resiliency for Regional Networks. Presented at 88th Annual Meeting of the Transportation Research Board, Washington, D.C., 2009.

4. Briguglio, L., G. Cordina, S. Bugeja, and N. Farrugia. Conceptualizing and Measuring Economic Resilience. 2005. https://secure.um.edu. mt/_data/assets/pdf_file/0013/44122/resilience_index.pdf. Accessed Sept. 29, 2010.

5. Huiping, L., S.J. Fernandez, and A. Ganguly. Racial Geography, Economic Growth, and Natural Disaster Resilience. Nov. 10, 2005. http://www.gri.msstate.edu/research/katrinalessons/Documents/ Resiliency\%20Manuscript.pdf. Accessed Sept. 29, 2010.

6. Bruneau, M., S. E. Chang, R. T. Eguchi, G.C. Lee, T.D. O'Rourke, A. M. Reinhorn, M. Shinozuka, K. Tierney, W. A. Wallace, and D. van Winterfeldt. A Framework to Qualitatively Assess and Enhance the Seismic Resilience of Communities. Earthquake Spectra, Vol. 19, No. 4, 2003, pp. 733-752.

7. Prevention web. City Profile: Climate and Disaster Resilience. 2009. http://www.preventionweb.net/files/8168_cityprofilelowresolution.pdf. Accessed Sept. 29, 2010.

8. Foster, H.D. Resilience Theory and System Evaluation. In Verification and Validation of Complex Systems: Human Factors Issues (J.A. Wise, V. D. Hopkin, and P. Stager, eds.), Springer-Verlag, Berlin, 1993, pp. 35-60.

9. Evaluation of the Systems' Available Redundancy to Compensate for Loss of Transportation Assets Resulting from Natural Disasters or Terrorist Attacks. Battelle, Jan. 10, 2007. http://ntl.bts.gov/ lib/33000/33400/33441/final_report/pdf/volume_3/technical_issue_ papers/paper4e_02.pdf. Accessed Sept. 29, 2010.

10. Litman, T. Evaluating Accessibility for Transportation Planning. Victoria Transport Policy Institute, Victoria, British Columbia, Canada, March 23, 2010. http://www.vtpi.org/access.pdf. Accessed Sept. 29, 2010.

11. Ta, C., A. V. Goodchild, and K. Pitera. Structuring a Definition of Resilience for the Freight Transportation System. In Transportation Research Record: Journal of the Transportation Research Board, No. 2097, Transportation Research Board of the National Academies, Washington, D.C., 2009, pp. 19-25.

12. Murray-Tuite, P.M. A Comparison of Transportation Network Resilience Under Simulated System Optimum and User Equilibrium Conditions. Proc., Winter Simulation Conference, IEEE, New York, 2006.

13. Urena Serulle, N., K. Heaslip, B. Brady, W. C. Louisell, and J. Collura. Resiliency of Transportation Network of Santo Domingo, Dominican Republic: Case Study. In Transportation Research Record: Journal of the Transportation Research Board, No. 2234, Transportation Research Board of the National Academies, Washington, D.C., 2011, pp. 22-30.

14. Victoria Transport Policy Institute. Evaluating Transportation Resilience: Evaluating the Transportation System's Ability to Accommodate Diverse, Variable and Unexpected Demands with Minimal Risk. Jan. 26, 2010. http://www.vtpi.org/tdm/tdm88.htm. Accessed July 22, 2010.

15. Tierney, K., and M. Bruneau. Conceptualizing and Measuring Resilience: A Key to Disaster Loss Reduction. TR News, No. 250, May-June 2007, pp. 14-17.

16. Stevanovic, A., and B. Nadimpalli. Seismic Vulnerability and Emergency Response Analyses of UDOT Lifelines. Upper Great Plains Institute, North Dakota State University, Fargo. April 2010. http://www.trb.org/ BridgesOtherStructures/Blurbs/Seismic_Vulnerability_and_Emergency_ Response_Analy_163437.aspx. Accessed Aug. 3, 2010.

17. City-Data.com. All Cities in Utah. 2009. http://www.city-data.com/city/ Utah.html. Accessed Aug. 18, 2010.

18. Highway Capacity Manual 2010. Transportation Research Board of the National Academies, Washington, D.C., 2010.

19. Utah Department of Transportation. PeMS 10.0. 2010. http://udot.pems. traffic.com. Accessed Sept. 1, 2010.

20. Texas Transportation Institute, Texas A\&M University Systems. Performance Measure Summary: Salt Lake City, UT. 2007. http://mobility. tamu.edu/ums/congestion_data/tables/salt_lake_city.pdf. Accessed Sept. 1, 2010.

21. Surface Transportation Policy Project. Transportation Costs and the American Dream. July 2003. http://www.transact.org/library/decoder/ american_dream.pdf. Accessed July 22, 2010.

22. Internal Revenue Service, U.S. Department of Treasury. IRS Announces 2010 Standard Mileage Rates. Dec. 3, 2009. http://www.irs.gov/newsroom/ article/ $0, \mathrm{id}=216048,00 . \mathrm{html}$. Accessed July 22, 2010.

23. Intelligent Transportation Systems, U.S. Department of Transportation. Ensure Redundancy of Critical Components in Transportation Support Systems to Be Used in Case of an Emergency. May 2004. http://www. itslessons.its.dot.gov/its/benecost.nsf/Lesson?OpenForm\&2267EC3D CC57FBB2852576C70059F1E3. Accessed July 22, 2010.

24. Rodrigue, J.-P. Graph Theory: Measures and Indices. 2010. http:// www.people.hofstra.edu/geotrans/eng/ch1en/meth1en/ch1m3en.html. Accessed July 22, 2010.

25. Clemm, A. Network Management Fundamentals: A Guide to Understanding How Network Management Technology Really Works. Cisco Press, Indianapolis, Ind., 2006.

26. Federal Emergency Management Agency, U.S. Department of Homeland Security. Emergency Response Action Steps. June 4, 2009. http:// www.fema.gov/plan/ehp/response.shtm. Accessed Aug. 3, 2010.

27. U.S. Army Corps of Engineers. Disaster Resource Contractor Registry. 2010. http://www.lrl.usace.army.mil/disasterequip. Accessed Aug. 3, 2010.

28. Burton, R.E., II. CCR Search. 2010. https://www.bpn.gov/CCRSearch/ results.aspx. Accessed Aug. 19, 2010.

The Critical Transportation Infrastructure Protection Committee peer-reviewed this paper. 